\title{
Uniformly stable preconditioned mixed boundary element method for low-frequency electromagnetic scattering
}

\section{Report}

Author(s):

Christiansen, Snorre H.

Publication date:

2002-09

Permanent link:

https://doi.org/10.3929/ethz-a-004447320

Rights / license:

In Copyright - Non-Commercial Use Permitted

Originally published in:

SAM Research Report 2002-17 


\title{
Uniformly stable preconditioned mixed boundary element method for low-frequency electromagnetic scattering
}

\author{
S.H. Christiansen
}

Research Report No. 2002-17

September 2002

Seminar für Angewandte Mathematik Eidgenössische Technische Hochschule CH-8092 Zürich

Switzerland 
Uniformly stable preconditioned mixed boundary element method for low-frequency electromagnetic scattering

\author{
S.H. Christiansen \\ Seminar für Angewandte Mathematik \\ Eidgenössische Technische Hochschule \\ CH-8092 Zürich \\ Switzerland
}

Research Report No. 2002-17 September 2002

\begin{abstract}
We propose a mixed boundary element discretization of the Electric Field Integral Equation for which we have an Inf-Sup condition which is uniform in both the mesh-width $h$ and the wavenumber $k$, for small enough $h$ and $k$. For this equation we construct a preconditioner such that the spectral condition number of the preconditioned system is also bounded independently of $k$ and $h$.
\end{abstract}




\section{The continuous problem}

Let $\Omega_{-}$be a bounded domain in $\mathbb{R}^{3}$ with a smooth boundary $\Gamma$. The exterior domain $\mathbb{R}^{3} \backslash\left(\Omega_{-} \cup \Gamma\right)$ is denoted $\Omega_{+}$and the outward normal on $\Gamma$ is denoted $n$. The tangential trace operator is denoted $\gamma_{\mathrm{T}}$ and the normal trace operator is denoted $\gamma_{\mathrm{n}}$.

Let $Z$ be a positive constant, called impedance. For each wavenumber $k>0$ the timeharmonic Maxwell equations (in any given open region of $\mathbb{R}^{3}$ ) are:

$$
\operatorname{curl} E=+i k Z H, \quad \operatorname{curl} H=-i k / Z E .
$$

Given a family $\left(E_{k}^{i n c}, H_{k}^{i n c}\right)$ for small positive $k$ of solutions of Maxwell's equations on a neighborhood of $\Gamma$ we are interested, for each $k$, in the solution $\left(E_{k}, H_{k}\right)$ of Maxwell's equations in $\Omega_{-}$or $\Omega_{+}$satisfying the perfect conductor boundary condition $\gamma_{\mathrm{T}} E_{k}=-\gamma_{\mathrm{T}} E_{k}^{\text {inc }}$, and (in the exterior domain) the Silver-Müller radiation condition.

We use potentials to represent $E_{k}$. Let $G_{k}$ denote the standard Green kernel of $-\Delta-k^{2}$ and let $\Phi_{k}$ be the single layer potential defined on scalar or tangent fields $u$ on $\Gamma$ by:

$$
\left(\Phi_{k} u\right)(y)=\int_{\Gamma} G_{k}(x, y) u(x) \mathrm{d} x, \quad G_{k}(x, y)=\frac{e^{i k|x-y|}}{4 \pi|x-y|} .
$$

We represent $E_{k}$ as an electric field generated by a tangent field $u_{k}$ on $\Gamma$ (the electric current). More precisely we put $E_{k}(y)=\left(\operatorname{grad} \operatorname{div}+k^{2}\right)\left(\Phi_{k} u_{k}\right)$. Letting $A_{k}=-\gamma_{\mathrm{T}}\left(\operatorname{grad} \operatorname{div}+k^{2}\right) \Phi_{k}$, the problem is to solve the Electric Field Integral Equation (EFIE) $A_{k} u_{k}=\gamma_{\mathrm{T}} E_{k}^{i n c}$.

The operator $A_{k}$ is continuous from $X=\mathrm{H}_{\text {div }}^{-1 / 2}(\Gamma)$ to its dual $X^{\prime}=\mathrm{H}_{\text {rot }}^{-1 / 2}(\Gamma)$, and the EFIE can be put in variational form:

$$
u_{k} \in X, \forall u^{\prime} \in X \quad\left\langle A_{k} u_{k}, u^{\prime}\right\rangle=\left\langle E_{k}^{i n c}, u^{\prime}\right\rangle .
$$

We denote by $a_{k}$ the associated bilinear form ; its expression on smooth fields is:

$$
a_{k}(u, v)=\iint_{\Gamma \times \Gamma} G_{k}(x, y)\left(\operatorname{div} u(x) \operatorname{div} v(y)-k^{2} u(x) \cdot v(y)\right) \mathrm{d} x \mathrm{~d} y
$$

Following Bendali [1] this variational problem is solved with the Galerkin method on divconforming Finite Element spaces on the boundary. At low frequencies one sees that the problem is that the limit of the operator $A_{k}$ as $k \rightarrow 0$, is degenerated ; in fact the limit is not even Fredholm since its kernel contains the infinite dimensional space $\operatorname{rot} \mathrm{H}^{1 / 2}(\Gamma)$.

The object of this paper is to compute approximations of $u_{k}$ in a stable way for small $k$.

\section{The continuous remedy}

For simplicity we suppose that $\Gamma$ is connected and simply connected. As remarked by DeLaBourdonnaye [2], if we put $V=\operatorname{grad} H^{3 / 2}(\Gamma)$ and $W=\operatorname{rot} \mathrm{H}^{1 / 2}(\Gamma)$, then $V$ and $W$ are closed in $X$ and we have the decomposition:

$$
X=V \oplus W .
$$


We put $S=\mathrm{H}^{1 / 2}(\Gamma)$, and for any space $Y$ of scalar fields on $\Gamma$ we put $Y^{\bullet}=\{u \in Y:\langle u, 1\rangle=0\}$.

Let $\Xi_{k}: V \times S^{\bullet} \rightarrow X$ denote the isomorphism defined by $\Xi_{k}(v, p)=v+k^{-1} \operatorname{rot} p$. The four blocks of the bilinear form $\tilde{a}_{k}$ on $V \times S^{\bullet}$ defined by $\tilde{a}_{k}\left((v, p),\left(v^{\prime}, p^{\prime}\right)\right)=a_{k}\left(\Xi_{k}(v, p), \Xi_{k}\left(v^{\prime}, p^{\prime}\right)\right)$, have the expression:

$$
\left(\begin{array}{rr}
\iint G_{k}(x, y)\left(\operatorname{div} v(x) \operatorname{div} v^{\prime}(y)-k^{2} v(x) \cdot v^{\prime}(y)\right) \mathrm{d} x \mathrm{~d} y & -k \iint G_{k}(x, y) v(x) \cdot \operatorname{rot} p^{\prime}(y) \mathrm{d} x \mathrm{~d} y \\
-k \iint G_{k}(x, y) \operatorname{rot} p(x) \cdot v^{\prime}(y) \mathrm{d} x \mathrm{~d} y & -\iint G_{k}(x, y) \operatorname{rot} p(x) \cdot \operatorname{rot} p^{\prime}(y) \mathrm{d} x \mathrm{~d} y
\end{array}\right)
$$

Since there is $C>0$ such that:

$$
\forall v \in V \quad\|v\|_{X} \leq C\|\operatorname{div} v\|_{\mathrm{H}^{-1 / 2}(\Gamma)}, \quad \forall p \in S^{\bullet} \quad\|p\|_{S} \leq C\|\operatorname{rot} p\|_{\mathrm{H}_{\mathrm{T}}^{-1 / 2}(\Gamma)},
$$

the two diagonal blocks are coercive hence invertible for $k=0$. We remark also that the coupling blocks vanish for $k=0$.

Concerning the right-hand sides we remark that:

$$
k^{-1}\left\langle\gamma_{\mathrm{T}} E_{k}^{i n c}, \operatorname{rot} p^{\prime}\right\rangle=i Z\left\langle\gamma_{\mathrm{n}} H_{k}^{i n c}, p^{\prime}\right\rangle .
$$

If for instance the family of incident waves consists of plane waves:

$$
E_{k}^{i n c}(x)=E_{0} e^{i k \sigma \cdot x}, \quad H_{k}^{i n c}(x)=1 / Z E_{0} \times \sigma e^{i k \sigma \cdot x},
$$

then the limit of $\gamma_{\mathrm{T}} E_{k}^{i n c}$ is a surface gradient and $\gamma_{\mathrm{n}} H_{k}^{i n c}$ has a non-zero limit. Thus both righthand sides in $V^{\star} \times S^{\bullet \star}$ have non-zero limits as $k \rightarrow 0$. It follows that with the decomposition $u_{k}=\Xi_{k}\left(v_{k}, p_{k}\right)$ both $v_{k}$ and $p_{k}$ have a non-zero limit as $k \rightarrow 0$.

We now turn to the preconditioning of the variational problem associated with (6) and we recall the remark made in [3] that a preconditioner is obtained by an invertible bilinear form on a dual space. Since the off-diagonal terms are small in norm and compact it is enough to precondition the two diagonal blocks.

For the first block, we proceed as follows. Put $V^{\prime}=\operatorname{grad} H^{1 / 2}(\Gamma)$. Then we remark that the $\mathrm{L}_{\mathrm{T}}^{2}(\Gamma)$-bilinear form extends continuously to an invertible bilinear form on $V^{\prime} \times V$. Let $\Theta_{1}: V^{\star} \rightarrow V^{\prime}$ be the corresponding isomorphism. We remark furthermore that $V^{\prime}$ is a closed subspace of $\mathrm{H}_{\mathrm{T}}^{-1 / 2}(\Gamma)$, hence we can use the bilinear form associated with the single layer operator on tangent fields as a preconditioner.

For the second block, the induced operator on $S^{\bullet} \rightarrow S^{\bullet \star}$ is the main part of the hypersingular operator appearing in acoustics. It can be efficiently preconditioned by the single layer operator [6] [3]. As a matter of notations we put $S^{\prime}=\mathrm{H}^{-1 / 2}(\Gamma)$ so that the $\mathrm{L}^{2}(\Gamma)$-bilinear form extends continuously to an invertible bilinear form on $S^{\bullet \bullet} \times S^{\bullet}$, and let $\Theta_{2}: S^{\bullet \star} \rightarrow S^{\prime \bullet}$ be the corresponding isomorphism.

Thus, on $V^{\prime} \times S^{\prime \bullet}$ we use the bilinear form $b$ whose block expression is:

$$
\left(\begin{array}{cc}
\iint G_{0}(x, y) v(x) \cdot v^{\prime}(y) \mathrm{d} x \mathrm{~d} y & 0 \\
0 & -\iint G_{0}(x, y) q(x) q^{\prime}(y) \mathrm{d} x \mathrm{~d} y
\end{array}\right)
$$

Letting $\Theta: V^{\star} \times S^{\bullet \star} \rightarrow V^{\prime} \times S^{\bullet \bullet}$ be the map componentwise induced by $\Theta_{1}$ and $\Theta_{2}$, and associating an operator $\tilde{\mathcal{A}}_{k}: V \times S^{\bullet} \rightarrow V^{\star} \times S^{\bullet \star}$ with $\tilde{a}_{k}$ and $\mathcal{B}: V^{\prime} \times S^{\prime \bullet} \rightarrow V^{\prime \star} \times S^{\prime \bullet \star}$ with $b$ we have: 
Proposition 2.1 There is $\epsilon>0$ such that for all $k \in[0, \epsilon]$ the operator $\Theta^{\star} \mathcal{B} \Theta \tilde{\mathcal{A}}_{k}$ is an automorphism of $V \times S^{\bullet}$ and all terms of the composition are isomorphisms whose norm and norm of the inverse are bounded independently of $k$ in $[0, \epsilon]$.

\section{Discretization}

Since $V=\operatorname{grad} \mathrm{H}^{3 / 2}(\Gamma)$ it would be cumbersome to implement a conforming Finite Element discretization of the variational problem on $V \times S^{\bullet}$. Instead we propose the following nonconforming method.

Suppose we have (finite dimensional) subspaces $X_{h}$ of $X \cap \mathrm{H}_{\mathrm{div}}^{0}(\Gamma)$ and $S_{h}$ of $S \cap \mathrm{H}^{1}(\Gamma)$, which are stable under complex conjugation, which are such that $S_{h}$ contains the constant fields and we have an exact sequence:

$$
S_{h} \stackrel{\operatorname{rot}}{\longrightarrow} X_{h} \stackrel{\operatorname{div}}{\longrightarrow} \mathrm{L}^{2}(\Gamma)
$$

We define $V_{h}$ by:

$$
V_{h}=\left\{u \in X_{h} \quad: \forall p \in S_{h} \quad\langle u, \operatorname{rot} p\rangle=0\right\} .
$$

We keep the notation $\tilde{a}_{k}$ to denote the extension of $\tilde{a}_{k}$ to $X \times S$ whose block-wise expression is given by (6). We solve the system: Find $\left(v_{k h}, p_{k h}\right) \in V_{h} \times S_{h}^{\bullet}$, such that for all $\left(v^{\prime}, p^{\prime}\right) \in V_{h} \times S_{h}^{\bullet}$ we have:

$$
\tilde{a}_{k}\left(\left(v_{k h}, p_{k h}\right),\left(v^{\prime}, p^{\prime}\right)\right)=\left\langle E_{k}^{i n c}, v^{\prime}\right\rangle+i Z\left\langle H_{k}^{i n c} \cdot n, p^{\prime}\right\rangle .
$$

Recall the definition of the gap : $\delta_{X}\left(V_{h}, V\right)=\sup _{v_{h} \in V_{h}} \inf _{v \in V}\left\|v_{h}-v\right\|_{X} /\left\|v_{h}\right\|_{X}$. Our first proposition concerns the well posedness of the discrete system.

Proposition 3.1 If $\delta_{X}\left(V_{h}, V\right) \rightarrow 0$ as $h \rightarrow 0$ then there is $\epsilon>0, h_{0}>0$ and $C>0$ such that for all $k \in[0, \epsilon]$, all $h<h_{0}$ we have :

$$
\inf _{(v, p) \in V_{h} \times S_{h}^{\bullet}} \sup _{\left(v^{\prime}, p^{\prime}\right) \in V_{h} \times S_{h}^{\bullet}} \frac{\left|\tilde{a}_{k}\left((v, p),\left(v^{\prime}, p^{\prime}\right)\right)\right|}{\|(v, p)\|_{X \times S}\left\|\left(v^{\prime}, p^{\prime}\right)\right\|_{X \times S}} \geq 1 / C .
$$

-Proof: Actually we prove uniform coercivity. By the continuity of the operators with respect to $k$ it suffices to prove coercivity for $k=0$. Let $P$ be the projector with range $V$ and kernel $W$. We have:

$$
\begin{aligned}
\forall v \in V_{h} \quad\|v\|_{X} & \leq\|v-P v\|_{X}+\|P v\|_{X} \\
& \leq\|I-P\| \delta\left(V_{h}, V\right)\|v\|+\|\operatorname{div} v\|_{\mathrm{H}^{-1 / 2}(\Gamma)}
\end{aligned}
$$

Therefore we have an estimate of the form : There is $h_{0}>0$ and $C>0$ such that for all $h<h_{0}$ :

$$
\forall v \in V_{h} \quad\|v\|_{X} \leq C\|\operatorname{div} v\|_{\mathrm{H}^{-1 / 2}(\Gamma)} .
$$

The result entails. 
In general we do not have a basis of $V_{h}$, hence solving this system requires some extra work. In our case this will be carried out by the preconditioner which we define now. It should be checked that in what follows only bases of $X_{h}$ and $S_{h}$ are needed.

Let $\Theta_{1 h}: X_{h}^{\star} \rightarrow X_{h}$ denote the map which to any $\ell \in X_{h}^{\star}$ associates the solution of:

$$
v \in V_{h}, \forall v^{\prime} \in V_{h} \quad\left\langle v, v^{\prime}\right\rangle=\ell\left(v^{\prime}\right) .
$$

For $\ell \in X_{h}^{\star}, \Theta_{1 h} \ell$ can be computed simply as the solution $u$ of:

$$
\begin{array}{r}
p \in S_{h}^{\bullet}, \forall p^{\prime} \in S_{h}^{\bullet} \quad\left\langle\operatorname{rot} p, \operatorname{rot} p^{\prime}\right\rangle=\ell\left(\operatorname{rot} p^{\prime}\right), \quad u \in X_{h}, \forall u^{\prime} \in X_{h} \\
\left\langle u, u^{\prime}\right\rangle=\ell\left(u^{\prime}\right)-\left\langle\operatorname{rot} p, u^{\prime}\right\rangle .
\end{array}
$$

We define the discretization $\Theta_{2 h}$ of $\Theta_{2}$ to be the map which to $\ell \in S_{h}^{\star}$ associates the solution $p$ of:

$$
p \in S_{h}^{\bullet}, \forall p^{\prime} \in S_{h}^{\bullet}\left\langle p, p^{\prime}\right\rangle=\ell\left(p^{\prime}\right) .
$$

Let $\Theta_{h}: X_{h}^{\star} \times S_{h}^{\star} \rightarrow X_{h} \times S_{h}$ be the association of $\Theta_{1 h}$ and $\Theta_{2 h}$. We keep the notation $b$ for the extension of $b$ from $V^{\prime} \times S^{\bullet}$ to $\mathrm{H}_{\mathrm{T}}^{-1 / 2}(\Gamma) \times S^{\prime}$ keeping the block expression (10). Let $\mathcal{B}_{h}: X_{h} \times S_{h} \rightarrow X_{h}^{\star} \times S_{h}^{\star}$ be the map induced by $b$. We also denote by $\tilde{\mathcal{A}}_{k h}$ the map induced by $\tilde{a}_{k}$ on $X_{h} \times S_{h} \rightarrow X_{h}^{\star} \times S_{h}^{\star}$.

One sees that the operator $\Theta_{h}^{\star} \mathcal{B}_{h} \Theta_{h}$ is a surjection onto $V_{h} \times S_{h}^{\bullet}$. For $\ell \in\left(X_{h} \times S_{h}^{\bullet}\right)^{\star}$, $\Theta_{h}^{\star} \mathcal{B}_{h} \Theta_{h} \ell$ depends only on $\left.\ell\right|_{V_{h} \times S_{h}^{\bullet}}$. It follows that the conjugate gradient algorithm for $\tilde{\mathcal{A}}_{k h}$ on $X_{h} \times S_{h}^{\bullet}$, preconditioned by $\Theta_{h}^{\star} \mathcal{B}_{h} \Theta_{h}$ yields iterates in $V_{h} \times S_{h}^{\bullet}$ converging to the solution of (13). Morevover $\Theta_{h}^{\star} \mathcal{B}_{h} \Theta_{h} \tilde{\mathcal{A}}_{k h}$ determines a bijection $V_{h} \times S_{h}^{\bullet} \rightarrow V_{h} \times S_{h}^{\bullet}$ whose spectral condition number $\kappa_{k h}$ is bounded independently of $k$ in an interval $[0, \epsilon]$.

More precise estimates on $\kappa_{k h}$ and the convergence of Krylov subspace methods, depend on the actual Galerkin spaces. Examples of Finite Element spaces which satisfy the above conditions include the case where we have quasi-uniform triangulations of $\Gamma$ and take for $X_{h}$ Raviart-Thomas vector FE of degree $n$ and for $S_{h}$ the scalar continuous piecewise $P^{n+1}$ FE. Then we also have the following stability property:

Proposition 3.2 There is $\epsilon>0, h_{0}>0$ and $C_{1}, C_{2}>0$ such that for all $k \in[0, \epsilon]$, all $h<h_{0}$ we have:

$$
\begin{aligned}
\left\|\Theta_{h}^{\star} \mathcal{B}_{h} \Theta_{h} \tilde{\mathcal{A}}_{k h}(u, p)\right\|_{0} \leq & C_{1}\|(u, p)\|_{0} \\
& \text { with }\|(u, p)\|_{0}^{2}=\|u\|_{\mathrm{H}_{\mathrm{div}}^{0}(\Gamma)}^{2}+\|p\|_{\mathrm{H}^{1}(\Gamma)}^{2}, \\
\left\|\Theta_{h}^{\star} \mathcal{B}_{h} \Theta_{h} \tilde{\mathcal{A}}_{k h}(u, p)\right\|_{-1 / 2} \geq & C_{2}^{-1}\|(u, p)\|_{-1 / 2} \\
& \text { with }\|(u, p)\|_{-1 / 2}^{2}=\|u\|_{\mathrm{H}_{\text {div }}^{-1 / 2}(\Gamma)}^{2}+\|p\|_{\mathrm{H}^{1 / 2}(\Gamma)}^{2} .
\end{aligned}
$$

-Proof: We show the proof for the case of fixed $k=0$ and then indicate how the proof extends to the the case of $k$ in an interval $[0, \epsilon]$.

At $k=0$ the operator $\tilde{\mathcal{A}}_{k h}$ decouples and we can study the action on $V_{h}$ and $S_{h}^{\bullet}$ separately.

(i) Proof of estimate (21). Let $P_{V_{h}}$ denote the $\mathrm{L}_{\mathrm{T}}^{2}(\Gamma)$-orthogonal projection onto $V_{h}$, and $P_{S_{h}}$ denote the $\mathrm{L}^{2}(\Gamma)$-orthogonal projection onto $S_{h}^{\bullet}$. Let $\mathfrak{S}$ and $\mathfrak{S}_{\mathrm{T}}$ denote the single layer operator acting on scalar and tangent fields respectively. 
The discrete operator $\Theta_{h}^{\star} \mathcal{B}_{h} \Theta_{h} \tilde{\mathcal{A}}_{k h}$ decouples into the two operators:

$$
V_{h} \subset \mathrm{H}_{\text {div }}^{0}(\Gamma) \stackrel{\operatorname{div}}{\longrightarrow} \mathrm{L}^{2}(\Gamma) \stackrel{\mathfrak{S}}{\longrightarrow} \mathrm{H}^{1}(\Gamma) \stackrel{\operatorname{grad}}{\longrightarrow} \mathrm{L}_{\mathrm{T}}^{2}(\Gamma) \stackrel{P_{V_{h}}}{\longrightarrow} V_{h} \stackrel{\mathfrak{S}_{\mathrm{T}}}{\longrightarrow} \mathrm{H}_{\mathrm{T}}^{1}(\Gamma) \stackrel{P_{V_{h}}}{\longrightarrow} V_{h},
$$

and:

$$
S_{h}^{\bullet} \subset \mathrm{H}^{1}(\Gamma) \stackrel{\text { rot }}{\longrightarrow} \mathrm{L}_{\mathrm{T}}^{2}(\Gamma) \stackrel{\mathfrak{S}_{\mathrm{T}}}{\longrightarrow} \mathrm{H}_{\mathrm{T}}^{1}(\Gamma) \stackrel{\text { rot }}{\longrightarrow} \mathrm{L}^{2}(\Gamma) \stackrel{P_{S_{h}}}{\longrightarrow} S_{h}^{\bullet} \stackrel{\mathfrak{S}}{\longrightarrow} \mathrm{H}^{1}(\Gamma) \stackrel{P_{S_{h}}}{\longrightarrow} S_{h}^{\bullet} .
$$

For the first operator it thus suffices to show that the last occurrence of $P_{V_{h}}$ satisfies an estimate of the form:

$$
\forall u \in \mathrm{H}_{\mathrm{T}}^{1}(\Gamma) \quad\left\|P_{V_{h}} u\right\|_{\mathrm{H}_{\mathrm{div}}^{0}(\Gamma)} \leq C\|u\|_{\mathrm{H}_{\mathrm{T}}^{1}(\Gamma)} .
$$

Let $P_{X_{h}}$ denote the $\mathrm{L}_{\mathrm{T}}^{2}$-orthogonal projection onto $X_{h}$ and let $\Pi_{h}$ denote the standard interpolator onto $X_{h}$ (interpolating the fluxes through the edges of the curved triangles). Using the fact that $V_{h}$ is $\mathrm{L}_{\mathrm{T}}^{2}$-orthogonal to the kernel of div on $X_{h}$, and standard inverse inequalities we have for any $u \in \mathrm{H}_{\mathrm{T}}^{1}(\Gamma)$ :

$$
\begin{aligned}
\left\|\operatorname{div} P_{V_{h}} u\right\|_{\mathrm{L}^{2}(\Gamma)} & =\left\|\operatorname{div} P_{X_{h}} u\right\|_{\mathrm{L}^{2}(\Gamma)} \\
& \leq\left\|\operatorname{div}\left(P_{X_{h}} u-\Pi_{h} u\right)\right\|_{\mathrm{L}^{2}(\Gamma)}+\left\|\operatorname{div} \Pi_{h} u\right\|_{\mathrm{L}^{2}(\Gamma)} \\
& \leq C h^{-1}\left\|\operatorname{div}\left(P_{X_{h}} u-\Pi_{h} u\right)\right\|_{\mathrm{H}^{-1}(\Gamma)}+\left\|\operatorname{div} \Pi_{h} u\right\|_{\mathrm{L}^{2}(\Gamma)} \\
& \leq C h^{-1}\left\|P_{X_{h}} u-\Pi_{h} u\right\|_{\mathrm{L}_{\mathrm{T}}^{2}(\Gamma)}+\left\|\operatorname{div} \Pi_{h} u\right\|_{\mathrm{L}^{2}(\Gamma)} \\
& \leq C h^{-1}\left\|u-\Pi_{h} u\right\|_{\mathrm{L}_{\mathrm{T}}^{2}(\Gamma)}+\left\|\operatorname{div} \Pi_{h} u\right\|_{\mathrm{L}^{2}(\Gamma)}
\end{aligned}
$$

The estimate (25) now follows from the well-known properties of $\Pi_{h}$.

For the second operator the $\mathrm{H}^{1}(\Gamma)$-stability follows from the well-known $\mathrm{H}^{1}(\Gamma)$-stability of the $\mathrm{L}^{2}(\Gamma)$-projector onto $S_{h}^{\bullet}$ (in the last occurrence of $P_{S_{h}^{\bullet}}$ ).

Thus estimate (21) is proved.

(ii) Proof of estimate (22). Since $\mathcal{B}_{h}$ and $\tilde{\mathcal{A}}_{0 h}$ are coercive on $V_{h} \times S_{h}^{\bullet}$ in the $\mathrm{H}_{\mathrm{T}}^{-1 / 2}(\Gamma) \times$ $\mathrm{H}^{-1 / 2}(\Gamma)$ and $\mathrm{H}_{\text {div }}^{-1 / 2}(\Gamma) \times \mathrm{H}^{1 / 2}(\Gamma)$ norms respectively it suffices to show that the $\mathrm{L}^{2}$-dualities used for the projections are uniformly (with respect to $h$ ) continuous on $V_{h}$ and $S_{h}^{\bullet}$ in these norms. For the $\mathrm{L}^{2}$-duality on $S_{h}$ in the $\mathrm{H}^{-1 / 2}(\Gamma) \times \mathrm{H}^{1 / 2}(\Gamma)$ norm this is trivial. For the case of $V_{h}$ we proceed as follows:

Let $P$ denote the projector with range $V$ and kernel $W$. It preserves the divergence. As we remarked in [4] we have an estimate of the form:

$$
\forall v \in V_{h} \quad\|v-P v\|_{\mathrm{L}_{\mathrm{T}}^{2}(\Gamma)} \leq C h\|\operatorname{div} v\|_{\mathrm{L}^{2}(\Gamma)} .
$$

For any $v, v^{\prime} \in V_{h}$ we have:

$$
\begin{aligned}
\left|\left\langle v, v^{\prime}\right\rangle\right| & \leq\left|\left\langle v-P v, v^{\prime}\right\rangle\right|+\left|\left\langle P v, v^{\prime}\right\rangle\right| \\
& \leq C\|v-P v\|_{\mathrm{H}_{\mathrm{div}}^{0}(\Gamma)}\left\|v^{\prime}\right\|_{\mathrm{H}_{\mathrm{rot}}^{-1}(\Gamma)}+\|P v\|_{\mathrm{H}_{\mathrm{T}}^{1 / 2}}\left\|v^{\prime}\right\|_{\mathrm{H}_{\mathrm{T}}^{-1 / 2}(\Gamma)} \\
& \leq C h\|\operatorname{div} v\|_{\mathrm{L}^{2}(\Gamma)}\left\|v^{\prime}\right\|_{\mathrm{L}_{\mathrm{T}}^{2}(\Gamma)}+C\|\operatorname{div} v\|_{\mathrm{H}^{-1 / 2}(\Gamma)}\left\|v^{\prime}\right\|_{\mathrm{H}_{\mathrm{T}}^{-1 / 2}(\Gamma)} \\
& \leq C\|\operatorname{div} v\|_{\mathrm{H}^{-1 / 2}(\Gamma)}\|\| v^{\prime} \|_{\mathrm{H}_{\mathrm{T}}^{-1 / 2}(\Gamma)} .
\end{aligned}
$$

This completes the proof of estimate (22). 
To extend the results to to $k \in[0, \epsilon]$ we simply remark that $\mathcal{B}_{h}$ and $\tilde{\mathcal{A}}_{k h}$ are uniformly continuous for the norms used in the proof of (21) and uniformly coercive for the norms used in the proof of $(22)$, with respect to $k$ in an interval $[0, \epsilon]$.

It follows that $\kappa_{k h}$ is bounded by $C_{1} C_{2}$ for $(k, h)$ in the range $\left.[0, \epsilon] \times\right] 0, h_{0}[$.

\section{References}

[1] A. Bendali : Numerical analysis of the exterior boundary value problem for the timeharmonic Maxwell equations by a boundary finite element method; Part 1: The continuous problem ; Math. Comp., Vol. 43, No. 167, p. 29-46, 1984. Part 2: The discrete problem; Math. Comp., Vol. 43, No. 167, p. 47-68, 1984.

[2] A. DelaBourdonnaye : Décomposition de $\mathrm{H}_{\mathrm{div}}^{-1 / 2}(\Gamma)$ et nature de l'opérateur de SteklovPoincaré du problème extérieur de l'électromagnétisme; C. R. Acad. Sci. Paris Sér. I Math., Vol. 316, No. 4, p. 369-372, 1993.

[3] S.H. Christiansen, J.-C. NÉDÉLeC : Des préconditionneurs pour la résolution numérique des équations intégrales de frontière de l'acoustique ; C. R. Acad. Sci. Paris Sér. I Math., Vol. 330, No. 7, p. 617-622, 2000.

[4] S.H. Christiansen, J.-C. NÉdÉLEC : Des préconditionneurs pour la résolution numérique des équations intégrales de frontière de l'électromagnétisme; C. R. Acad. Sci. Paris, Sér. I Math., Vol. 331, No. 9, p. 733-738, 2000.

[5] J.-C. NÉdÉLEC : Acoustic and Electromagnetic Equations, Integral Representations for Harmonic Problems ; Springer-Verlag, 2001.

[6] O. Steinbach, W.L. Wendland : The construction of some efficient preconditioners in the boundary element method; Adv. Comput. Math., Vol. 9, No. 1-2, p. 191-216, 1998. 